\title{
Streptococcus bovis endocarditis as a presenting manifestation of idiopathic ulcerative colitis
}

\author{
M. Moshkowitz, N. Arber, R. Wajsman, M. Baratz ${ }^{1}$ and T. Gilat \\ Departments of Gastroenterology and ${ }^{1}$ Pathology, Ichilov Hospital, Tel-Aviv Medical Center, and the \\ Sackler Faculty of Medicine, Tel-Aviv University, Tel-Aviv, Israel
}

\begin{abstract}
Summary: Streptococcus bovis bacteraemia and endocarditis have been associated with several gastrointestinal diseases, mainly malignant or potentially malignant tumours, and less commonly non-malignant gastrointestinal disorders. We describe a 73 year old man in whom Streptococcus bovis endocarditis developed, and was the presenting manifestation of undiagnosed quiescent ulcerative colitis. Such an association has not been described previously.
\end{abstract}

\section{Introduction}

Streptococcus bovis is a normal inhabitant of the intestinal tract, and may be isolated in $5-16 \%$ of faecal samples in normal adults. ${ }^{1}$ The association of $S$. bovis with endocarditis has been known for more than 40 years. ${ }^{2}$ Since then, there have been many reports associating $S$. bovis endocarditis with carcinoma of the colon, ${ }^{3}$ and other colonic disorders including benign colonic polyps, caecal volvulus, colorectal diverticulae, perirectal abscess, and rectal manipulations. ${ }^{4-6}$ In some of these cases, the endocarditis presented before any symptoms of the colonic lesions had become evident. This report describes a 73 year old man, in whom $S$. bovis endocarditis developed, and was the presenting manifestation of undiagnosed quiescent ulcerative colitis.

\section{Case report}

A 73 year old man was admitted to the hospital with a 3 week history of intermittent fever, chills, general malaise and loss of appetite. Ten years earlier, the patient underwent aortic valve replacement with a biological valve due to aortic stenosis. Several months before the operation, an impairment of liver function tests was observed and a liver biopsy revealed chronic active hepatitis with early changes of cirrhosis. Two years prior to the present hospitalization he was seen with symptoms suggestive of irritable bowel with occasional minimal flecks of blood. A colonoscopy to $90 \mathrm{~cm}$ showed a

Correspondence: M. Moshkowitz, M.D., Department of Gastroenterology, Ichilov Hospital, 6 Weizman Street, Tel-Aviv 64239, Israel.

Accepted: 30 March 1992 spastic sigmoid, and a reddened, non-bleeding rectal mucosa. Biopsies of the sigmoid were normal. Rectal biopsies showed mild inflammation compatible with non-specific colitis. The patient was not treated as he was apparently well without any gastrointestinal complaints until 3 weeks before the present hospitalization.

On physical examination, he had a temperature of $38.2^{\circ} \mathrm{C}$, blood pressure of $110 / 60 \mathrm{mmHg}$, and grade $3 / 6$ systolic murmur over the precordium radiating to the neck. Investigation showed the haemoglobin was $11.9 \mathrm{~g} / \mathrm{dl}$ and ESR $58 \mathrm{~mm} /$ hour. White blood cell count $8,500 / \mathrm{mm}^{3}$ with a shift to the left, and platelet count $56,000 / \mathrm{mm}^{3}$. Plasma albumin $29 \mathrm{~g} / 1$, globulin $40 \mathrm{~g} / 1$, liver enzymes slightly raised, renal function normal. Urine had no red cells or proteinurea and stool no ova or parasites. Nine blood cultures grew $S$. bovis. Electrocardiogram showed normal sinus rhythm, and echocardiography showed a slightly enlarged, well-contracting left ventricle and artificial aortic valve with no evidence of vegetations on the valves.

Although the echocardiography was not diagnostic, the subacute course of the diseases, and the clinical and laboratory findings were all compatible with infective endocarditis. The patient was treated with intravenous ampicillin and gentamicin for 4 weeks, and recovered uneventfully.

Colonoscopy revealed areas of oedematous, friable mucosa along the transverse colon which bled easily on contact, and to a lesser degree in the right colon. The left colonic mucosa appeared normal. Biopsies were taken only from the inflamed regions and revealed an irregular surface lined by a columnar to cuboidal epithelium permeated by isolated polymorphs. There was loss and distortion of the crypts with a mainly good preservation of the goblet cell population lining the 
crypts. The stroma contained a large number of chronic inflammatory cells. In addition, there were polymorphonuclear eosinophils and neutrophils penetrating the lining epithelium of isolated crypts, and accumulating in the lumen of some, that is, cryptitis and crypt abscess (Figure 1). No granulomas and no amoebae were found in these biopsies. The histological features were compatible with active ulcerative colitis. The segmental distribution of the inflammatory process in the transverse and right colon with sparing of the left side, suggested the diagnosis of Crohn's colitis. Small bowel Xrays were normal. In view of the previous biopsy finding in the rectum, the diagnosis of ulcerative colitis seemed more likely. The patient was well on follow-up one year after hospitalization.

\section{Discussion}

Despite the report of Klein et al. ${ }^{3}$ that Streptococcus bovis is more frequently isolated from faeces of patients with inflammatory bowel disease, there are no reports on the association of $S$. bovis bacteraemia and endocarditis with inflammatory bowel disease. There is a single case report in the English language publications of Streptococcus faecalis endocarditis in association with Crohn's disease.? In that case, bacteraemia and aortic valve endocarditis developed during an exacerbation of the disease which was treated with immunosuppressive drugs, including prednisolone and azathioprine. In contrast, in the present case, the patient was asymptomatic and was not known to suffer from

\section{References}

1. Noble, C.J. Carriage of group D streptococci in the human bowel. J Clin Pathol 1978, 31: 1182-1186.

2. MacNeal, W.J. \& Blevins, A. Bacteriological studies in endocarditis. J Bacteriol 1945, 49: 603-610.

3. Klein, R.S., Recco, R.A., Catalano, M.D., Edberg, S.C., Casey, J.I. \& Steigbigel, N.H. Association of Streptococcus bovis with carcinoma of the colon. N Engl J Med 1977, 297: 800-802.

4. Friedrich, I.A., Wormser, G.P. \& Gottfied, E.B. The association of remote Streptococcus bovis bacteremia with colonic neoplasia. Am J Gastroenterol 1982, 77: 82-84.

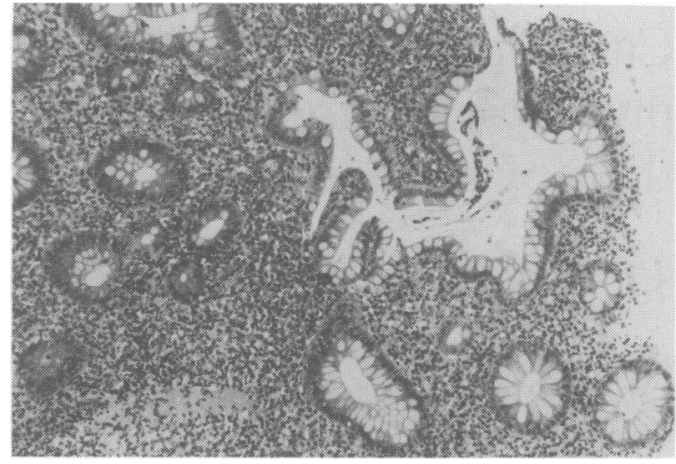

Figure 1 Active, ulcerative colitis with cryptitis, crypt abscess and a dense chronic and acute inflammatory infiltrate of the stroma $(H \& G \times 350)$.

inflammatory bowel disease. $S$. bovis bacteraemia and endocarditis were the presenting manifestations which led to the definite diagnosis of the intestinal disease. Liver disease and liver biopsy have also been associated with bacteraemia, ${ }^{8}$ but the liver disease in our patient was silent for many years, with no signs of exacerbation such as ascites or worsening of liver function tests at the time of the valve infection. It seems therefore that the source of the $S$. bovis infection was the inflammed, erosive mucosa of the large bowel. This case demonstrates the possibility of inflammatory bowel disease as a source of infection in $S$. bovis endocarditis.

5. Murray, H.W. \& Roberts, R.B. Streptococcus bovis bacteremia and underlying gastrointestinal diseases. Arch Intern Med 1978, 138: $1097-1099$

6. Bayliss, R. Clarke, S., Oakley, C.M., Somerville, W., Whitfield, A.G. \& Young, S.E.J. The bowel, the genitourinary tract, and infective endocarditis. Br Heart J 1984, 51: 339- 345 .

7. Wong, J.S. Infective endocarditis in Crohn's disease. Br Heart $J$ 1989, 62: 163-164.

8. McCloskey, R.V., Gold, M. \& Weser, E. Bacteremia after liver biopsy. Arch Intern Med 1973, 132: 213-215. 